\title{
Editorial: Key Electrochemical Energy Reactions Catalyzed by Nanomaterials
}

\author{
Tianyi Ma ${ }^{1 *}$, Lei Zhang ${ }^{2 *}$ and Zhenhai Wen ${ }^{3 *}$ \\ ${ }^{1}$ Discipline of Chemistry, University of Newcastle, Callaghan, NSW, Australia, ${ }^{2}$ School of Chemistry and Chemical \\ Engineering, South China University of Technology, Guangzhou, China, ${ }^{3}$ Fujian Institute of Research on the Structure of \\ Matter, Chinese Academy of Sciences, Fuzhou, China
}

Keywords: renewable energy, electrocatalysis, photocatalysis, nanomaterials, surface chemistry

\section{Editorial on the Research Topic}

\section{Key Electrochemical Energy Reactions Catalyzed by Nanomaterials}

The application for (photo)electrochemical technologies is set to expand rapidly in the recent several decades as demand grows in the environment protection, clean energy storage, and supply. Several unique characteristics of functionalized nanostructured materials or transition metal-nitrogen-carbon complex make them ideal catalyst candidates for combining high energy and power at the material level. These advantages include: (i) increased photo/electrochemically active surface areas for charge transfer, (ii) reduction of photo/electronic and ionic transport resistance at smaller diffusion length scales, and (iii) the ability to incorporate high-energy materials into a nanostructured framework capable of sustaining high powers (Lee et al., 2011; Seh et al., 2017; Sagar et al., 2018).

Recent advancement of nanostructured materials or transition metal-nitrogen-carbon complex

Edited and reviewed by:

Climent Victor,

University of Alicante, Spain

*Correspondence:

Tianyi Ma

tianyi.ma@newcastle.edu.au

Lei Zhang

celeizhang@scut.edu.cn

Zhenhai Wen

wen@fjirsm.ac.cn

Specialty section:

This article was submitted to Catalysis and Photocatalysis,

a section of the journal

Frontiers in Chemistry

Received: 16 September 2019 Accepted: 06 December 2019 Published: 20 December 2019

Citation:

Ma T, Zhang $L$ and Wen $Z$ (2019) Editorial: Key Electrochemical Energy Reactions Catalyzed by Nanomaterials. Front. Chem. 7:881. doi: 10.3389/fchem.2019.00881 has improved (photo)electrochemical performance significantly, especially in term of catalysis, including but not limited to oxygen reduction reaction (ORR), oxygen evolution reaction (OER), hydrogen evolution reaction (HER), nitrogen reduction reaction (NRR), and carbon dioxide reduction reaction (CRR). Among them, the noble metal-based catalysts exhibit attractive catalytic performance, but their rare nature hinders large-scale application. In recent years, a large variety of non-noble-metal or non-metal-based alternative catalysts using abundant and low-cost $3 \mathrm{~d}$ metals (e.g., Fe, Co) has been studied with increasing activity and stability (Yan et al., 2019).

As future applications, besides considering the low cost, a major challenge will be to reduce and bridge the performance gap by combining high specific surface active sites, high photo/electronic conductivity and good mechanical and chemical stability. In this Research Topic, we present a highlighted collection of original research articles that show how transition metal-nitrogen-carbon complex and nanomaterials such as $2 \mathrm{D}$ transition metal nanofilms, hollow metal microspheres, and graphene offer opportunities to develop potentially novel material structures that can reach these goals.

Recently, it has been indicated that developing cheap, highly efficient, and stable bifunctional electrocatalysts for both hydrogen and oxygen evolution reactions (HER and OER) has garnered a great interest with studies showing their encouraging large-scale application of water splitting technology. Zhang et al. reported novel $\mathrm{CoS}_{2}$ nanoparticles supported on nitrogen-doped graphene $\left(\mathrm{CoS}_{2} @ \mathrm{~N}-\mathrm{GN}\right)$ by a one-step hydrothermal method; the resultant hybrid exhibited remarkable overall electrocatalytic activity toward OER and HER in the alkaline electrolyte with enhanced long-term stability. Moreover, attributed to the high intrinsic activity of $\mathrm{CoS}_{2}$ nanocrystals, efficient electron transfer provided by $\mathrm{N}$-doped graphene and the synergetic coupling interaction between 
two components, the $\mathrm{CoS}_{2} @ \mathrm{~N}-\mathrm{GN}$ enabled the assembled water splitting device with low cell voltage, high efficiency, and prolonged operational life, which would provide insight into the rational design of transition metal chalcogenides for highly efficient and durable hydrogen and oxygen-involved electrocatalysis. On the other hand, implanting noble metal onto transition metal-nanofilms as one of the significant strategies has been shown to greatly control and increase the catalytic activity toward the ORR performance. More specifically, Pdbased electrocatalysts show excellent stability in alkaline solution owing to a less corrosive environment, while Co-decorated catalysts have the advantages of their low cost and promising applications in oxygen electrode (Cheng et al., 2017a,b). As a demonstration, Pd nanoparticles implanted onto Co nanofilms (Pdx/Co-nanofilms/C) were synthesized on an immiscible ionic liquid (IL)/water interface by $\mathrm{An}$ et al. Due to a marked distortion of crystal lattice and surface roughness showing their larger catalytic areas, the $\mathrm{Pdx} / \mathrm{Co}$-nanofilms/C catalysts exhibited enhanced ORR catalytic activity in both acid and alkaline media. In conjunction, the designed catalysts exhibited superior stability in alkaline media after using a proper heat-treatment method. Besides, with the advantages of alkaline fuel cells increasingly prominent due to the emergence, development and application of anion exchange membranes, ORR is also an important part of the alkaline fuel cell (Lefevre et al., 2009; Wang et al., 2012; Shen et al., 2018). Among the non-precious metal catalysts, the transition metal-nitrogen-carbon complex $(\mathrm{M}-\mathrm{N}-\mathrm{C})$ as the most attractive candidate, has achieved landmark achievements and might be commercially employed in fuel cells in the near future (Artyushkova et al., 2015; Kim et al., 2017; Li et al., 2017). Thus, $\mathrm{Gu}$ et al. showed a nitrogen-rich ligand PIPhen as a precursor that was reacted with $\mathrm{Fe}^{2+}$ to form the coordination polymer on the carbon powder (Fe-PIPhen/C). The prepared Fe-PIPhen/C catalyst possessed high ORR activity, only slightly lower than that of the $\mathrm{Pt} / \mathrm{C}$ catalyst. Without employing the pyrolysis process, the $\mathrm{Fe}-\mathrm{PIPhen} / \mathrm{C}$ catalyst has advantages in low cost and controllable structures, being promising alternatives to the $\mathrm{Pt} / \mathrm{C}$ catalyst in the fuel cell ORR process.

Although nanostructured materials with a variety of useful functionalities are widely applied in electrocatalysis and energy storage (Guo et al., 2017), some environmental-friendly magnetic materials as important photocatalysts are also potential for a wide range of practical applications (Ma et al., 2018), such as hematite $\left(\alpha-\mathrm{Fe}_{2} \mathrm{O}_{3}\right)$. Since self-assembled hematite with highly specific hollow nano/micro-structures and unique properties have emerged as being of great interest to material scientists, porous self-assembled $\alpha-\mathrm{Fe}_{2} \mathrm{O}_{3}$ hollow microspheres were successfully synthesized by Yin et al. via a simple and feasible IL-assisted solvothermal method. In this work, the IL $\left[\mathrm{C}_{4} \mathrm{Mim}\right] \mathrm{BF}_{4}$ was used

\section{REFERENCES}

Artyushkova, K., Serov, A., Rojas-Carbonell, S., and Atanassov, P. (2015). Chemistry of multitudinous active sites for oxygen reduction reaction in transition metal-nitrogen-carbon electrocatalysts. J. Phys. Chem. C 119, 25917-25928. doi: 10.1021/acs.jpcc.5b07653 not only as solvents, but also as templates for the formation of porous hollow spheres with improved properties (Endres et al., 2003; Cooper et al., 2004; Liu et al., 2006). Attributed to the self-assembled structure and higher surface area, the as-synthesized $\alpha-\mathrm{Fe}_{2} \mathrm{O}_{3}$ hollow spheres exhibited excellent photocatalytic activity in Rhodamine $\mathrm{B}(\mathrm{RhB})$ photodegradation. Additionally, the main formation mechanism of the porous hollow structures has been proved to be a nucleation-aggregation evacuation; and the as-prepared photocatalysts can be recycled easily due to the ferromagnetic properties. As knowing that the high recombination rates of photogenerated electron-holes inhibit the catalytic activity of semiconductor photocatalyst, $\mathrm{Wu}$ et al. introduced a simple hydrothermal method to synthesize the heterojunctions of flower-like g- $\mathrm{C}_{3} \mathrm{~N}_{4} / \mathrm{BiOBr}$ composites as photocatalysts. BiOBr-g- $\mathrm{C}_{3} \mathrm{~N}_{4}$ (4:1) showed the most excellent properties of photocatalytic degradation bisphenol (BPA) under the visible light due to its narrower bandgap than that of pure $\mathrm{BiOBr}$. Moreover, the separation of photogenerated carriers can be facilitated by the heterostructure between $\mathrm{BiOBr}$ and $\mathrm{g}-\mathrm{C}_{3} \mathrm{~N}_{4}$.

We believe that the Key Electrochemical Energy Reactions Catalyzed by Nanomaterials Research Topic exemplifies various nanomaterials research development in the application of (photo)electrocatalysis and provides research strategies on how to improve these catalytic performances. The (photo)electrocatalysis plays a central role in the environment, clean energy conversion, enabling sustainable processes for future technologies (Seh et al., 2017). Thus, the Research Topic aims to know the research frontier of Energy Reactions Catalysts, with faster and more efficient methods to capture this understanding from these more advanced researches, which can further guide the community to seek for high-performance catalysts.

\section{AUTHOR CONTRIBUTIONS}

All authors listed have made a substantial, direct and intellectual contribution to the work, and approved it for publication.

\section{FUNDING}

TM is a senior lecturer and ARC DECRA fellow (DE150101306). He acknowledges Faculty of Science Strategic Investment Funding 2019 of University of Newcastle and CSIRO. LZ is a professor of South China University of Technology, acknowledges National Key Research and Development Program of China (2016YFA0202604). ZW is a professor of Fujian Institute of Research on the Structure of Matter, Chinese Academy of Sciences, Fuzhou. He acknowledges the National Natural Science Foundation of China (Project No. 21875253). et al. (2017a). A modified molecular framework derived highly efficient Mn-Cocarbon cathode for flexible Zn-air battery. Chem. Commun. 53, 11596-11599. doi: 10.1039/c7cc04099g

Cheng, H., Li, M. L., Su, C. Y., Li, N., and Liu, Z. Q. (2017b). Cu-Co bimetallic oxide quantum dot decorated nitrogen-doped carbon nanotubes: a 
highefficiency bifunctional oxygen electrode for $\mathrm{Zn}$-air batteries. Adv. Funct. Mater. 27:1701833. doi: 10.1002/adfm.201701833

Cooper, E. R., Andrews, C. D., Wheatley, P. S., Webb, P. B., Wormald, P., and Morris, R. E. (2004). Ionic liquids and eutectic mixtures as solvent and template in synthesis of zeolite analogues. Nature 430:1012. doi: 10.1038/nature02860

Endres, F., Bukowski, M., Hempelmann, R., and Natter, H. (2003). Electrodeposition of nanocrystalline metals and alloys from ionic liquids. Angew. Chem. Int. Ed. 42, 3428-3430. doi: 10.1002/anie.200350912

Guo, X., Zheng, S., Zhang, G., Xiao, X., Li, X., Xu, Y., et al. (2017). Nanostructured graphene-based materials for flexible energy storage. Energy Storage Mater 9, 150-169. doi: 10.1016/j.ensm.2017.07.006

Kim, J. H., Sa, Y. J., Jeong, H. Y., and Joo, S. H. (2017). Roles of Fe-Nx and FeFe3C@C species in Fe-N/C electrocatalysts for oxygen reduction reaction. ACS Appl. Mater. Interfaces 9, 9567-9575. doi: 10.1021/acsami.6b13417

Lee, S. W., Gallant, B. M., Byon, H. R., Hammond, P. T., and Shao-Horn, Y. (2011). Nanostructured carbon-based electrodes: bridging the gap between thin-film lithium-ion batteries and electrochemical capacitors. Energy Environ. Sci. 4, 1972-1985. doi: 10.1039/c0ee00642b

Lefevre, M., Proietti, E., Jaouen, F., and Dodelet, J. P. (2009). Iron-based catalysts with improved oxygen reduction activity in polymer electrolyte fuel cells. Science 324:71. doi: 10.1126/science.1170051

Li, J. S., Chen, J. J., Wang, H. Y., Ren, Y., Liu, K., Tang, Y. G., et al. (2017). $\mathrm{Fe} / \mathrm{N}$ co-doped carbon materials with controllable structure as highly efficient electrocatalysts for oxygen reduction reaction in Al-air batteries. Energy Storage Mater. 8, 49-58. doi: 10.1016/j.ensm.2017.03.007

Liu, D. P., Li, G. D., Su, Y., and Chen, J. S. (2006). Highly luminescent $\mathrm{ZnO}$ nanocrystals stabilized by ionic-liquid components. Angew. Chem. 118, 7530-7533. doi: 10.1002/ange.200602429

Ma, J., Guo, X., Yan, Y., Xue, H., and Pang, H. (2018). FeO $\mathrm{F}_{\mathrm{x}}$-based materials for electrochemical energy storage. Adv. Sci. 5:1700986. doi: $10.1002 /$ advs. 201700986
Sagar, G., Soumik, D., Simran, K., Harish, R. I., Ashwani, K. T., and Venkataramanan, M. (2018). Effect of intrinsic properties of anions on the electrocatalytic activity of $\mathrm{NiCo}_{2} \mathrm{O}_{4}$ and $\mathrm{NiCo}_{2} \mathrm{O}_{\mathrm{x}} \mathrm{S}_{4}$-x grown by chemical bath deposition. ACS Omega 3, 9066-9074. doi: 10.1021/acsomega.8b 00952

Seh, Z. W., Kibsgaard, J., Dickens, C. F., Chorkendorff, I., Nørskov, J. K., and Jaramillo, T. F. (2017). Combining theory and experiment in electrocatalysis: insights into materials design. Science 355:146. doi: 10.1126/science.a $\operatorname{ad} 4998$

Shen, Y. J., Zhu, Y. L., Sunarso, J., Guan, D. Q., Liu, B., Liu, H., et al. (2018). Novel phosphorus-doped perovskite oxide as an oxygen reduction reaction electrocatalyst in an alkaline solution. Chem. Eur. J. 24, 6950-6957. doi: 10.1002/chem. 201705675

Wang, C., Markovic, N. M., and Stamenkovic, V. R. (2012). Advanced platinum alloy electrocatalysts for the oxygen reduction reaction. ACS Catal. 2, 891-898. doi: $10.1021 / \mathrm{cs} 3000792$

Yan, J. Q., Kong, L. Q., Ji, Y. J., White, J., Li, Y. Y., Zhang, J., et al. (2019). Single atom tungsten doped ultrathin $\alpha-\mathrm{Ni}(\mathrm{OH})_{2}$ for enhanced electrocatalytic water oxidation. Nat. Commun. 10:2149. doi: 10.10138/s41467-019-0 9845-z

Conflict of Interest: The authors declare that the research was conducted in the absence of any commercial or financial relationships that could be construed as a potential conflict of interest.

Copyright $\odot 2019 \mathrm{Ma}$, Zhang and Wen. This is an open-access article distributed under the terms of the Creative Commons Attribution License (CC BY). The use, distribution or reproduction in other forums is permitted, provided the original author(s) and the copyright owner(s) are credited and that the original publication in this journal is cited, in accordance with accepted academic practice. No use, distribution or reproduction is permitted which does not comply with these terms. 